\title{
Invited Commentary: Frontiers in Neurolaryngology
}

\author{
Michael M. Johns • Allen D. Hillel • \\ Adam M. Klein
}

Published online: 15 June 2013

(C) Springer Science+Business Media New York 2013

\begin{abstract}
Neurolaryngology is perhaps the most complex and poorly understood area of laryngology. Laryngologists largely treat neurological disorders of the larynx at the end organ level through static and symptom based manipulations. Interest in laryngology as a subspecialty of otolaryngology is rising with growth in formal clinical training programs, collaboration with neurology, neurosciences, and speech language pathology. This growth is creating a critical mass of individuals committed to narrowing the knowledge gap in neurolaryngology that presently limits optimum intervention for neurolaryngological disorders. An immediate and tangible frontier is the expansion and role of laryngeal electromyography in the field. Understanding laryngeal motor and sensory function peripherally and centrally over time will allow for dynamic and motion specific reanimation of vocal fold paralysis. The role of neural dysfunction in age-related voice change will help clarify the spectrum of disease in older patients and lead to functional therapies. Hyperfunctional disorders of the larynx, such as spasmodic dysphonia, vocal tremor, primary muscle tension dysphonia and paradoxical vocal fold
\end{abstract}

M. M. Johns $(\varangle)$ · A. M. Klein

Emory Voice Center, Emory University, 550 Peachtree St.

9th Fl. Ste 4400, Atlanta, GA 30342, USA

e-mail: michael.johns2@emory.edu

A. M. Klein

e-mail: Aklein4@emory.edu

M. M. Johns

Otolaryngology-Head and Neck Surgery, Emory University,

550 Peachtree St. 9th Fl. Ste 4400, Atlanta, GA 30342, USA

A. D. Hillel

Otolaryngology - Head and Neck Surgery, University

of Washington, PO Box 356515, Seattle, WA 98195-6515, USA

e-mail: ahillel@u.washington.edu dysfunction will be more precisely diagnosed with targeted intervention at the source. Understanding, diagnosing, and treating sensory dysfunction of the larynx remains a final frontier with implications not only for disorders such as laryngeal sensory neuropathic cough, but also disorders that have been traditionally thought of as primary motor problems, such as spasmodic dysphonia. Finally, improved understanding of the afferent neural supply to the larynx will aid in the site-specific treatment of neurogenic cough and irritable larynx syndrome.

Keywords Larynx $\cdot$ Neurolaryngology $\cdot$ Voice . Laryngeal EMG

\section{Introduction}

The field of laryngology has been rapidly changing over the past half century. Advances in understanding of laryngeal physiology and pathology combined with advanced technology has led to novel diagnostic and therapeutic techniques to treat patients with voice, swallowing, and airway disorders. Neurolaryngology may be the most complex area of this field and much work remains to elucidate the complex interaction of central neural function interfacing with the tissues of the larynx and pharynx.

Frontiers in our understanding of neurolaryngeal physiology and pathology are being pushed and great advances are forthcoming over the next several decades. The authors anticipate that there will be a shift from speculation to concrete understanding about neurolaryngeal function and dysfunction. As concrete understanding unfolds, laryngologists will shift from treating the end organ of the larynx and pharynx to the source of the pathology. The result will be improved ability to intervene in hyperfunctional 
neurolaryngeal disorders such as unilateral vocal fold paralysis (UVFP) and paresis, bilateral vocal fold paralysis (BVFP), and the aging voice, hyperfunctional disorders including laryngeal dystonia, tremor, primary muscle tension dysphonia (MTD), paradoxical vocal fold dysfunction (PFVF) and poorly understood sensory disorders of the larynx.

This issue of Current Otorhinolaryngology Reports highlights four contemporary topics in neurolaryngology: Vocal fold paresis, the state of the art in laryngeal EMG, evaluation and management of acute recurrent laryngeal nerve injury, and the clinical implications of the laryngeal adductor reflex. These topics reveal the state of the art in neurolaryngology and point out knowledge gaps that will help drive future research.

\section{The Impact of Training in Laryngology}

The rising of interest in advanced training in laryngology has led to a growth in fellowship training programs. Coupled with multidisciplinary interaction with colleagues in speechlanguage pathology, voice science, neurology and neurosciences, a critical mass of expertise and interest in laryngeal disease will push frontiers in laryngology forward.

One concrete example in neurolaryngology is the increasing prevalence of the use of diagnostic laryngeal electromyography (LEMG). Though our understanding of LEMG is nascent, pioneers in our field are exploring new ways to utilize this technology in a refined way to understand laryngeal pathophysiology. Fine wire electromyography allows a greater ability to study laryngeal function in a precise way freely during laryngeal motor tasks. Combined with advances in quantitative LEMG, this technique will shift from being a fairly subjective assessment to an objective diagnostic tool.

As this frontier is pushed, the first application of LEMG will likely be in UVFP. There will be a shift from a primarily "wait and see" approach in UVFP to active diagnosis and directed treatment of recurrent and superior laryngeal nerve deficits. As this occurs, LEMG will be a staple in the laryngologists practice and the use of LEMG will grow further in other motor disorders of the larynx. Early research in electrodiagnostic sensory testing in the larynx shows promise for quantifying sensory dysfunction. This will have significant impact in diagnosing and treating not only dysphagia, but also disorders that may have complex sensory/motor interactions such as spasmodic dysphonia, PFVF, sensory neuropathic cough, primary MTD, and laryngeal manifestations of systemic neurologic conditions.

\section{Frontiers in Knowledge, Diagnosis and Treatment}

Globally, advances in knowledge of the complex peripheral sensory and motor innervation of the larynx, central control of laryngeal musculature and sensory feedback, and the interface between the two will help elucidate the pathophysiology of neurolaryngeal disease. In particular, further understanding of denervation, reinnervation, and synkinesis in UVFP/BVFP will be a platform to modify functional outcomes. With more clarity on the natural history of denervation and reinnervation, systemic medical therapies such as nimodipine, and local administration of growth factors and agents to promote favorable reinnervation will become mainstay in at least the acute setting. Furthermore, interventions for UVFP, such as laryngeal framework surgery and present reinnervation strategies, remain static geometric solutions to a dynamic problem. Motion specific reanimation of UVFP is on the horizon possibly using novel reinnervation techniques like muscle-nerve-muscle neurotization and electrical pacing modalities.

Age related voice changes remain fairly poorly understood. Though knowledge of end organ tissue changes is advancing, the neurolaryngological elements of this disorder are not well understood. There is a wide spectrum of age related changes in vocal function that may have roots partly in chronic denervation and reinnervation. Refining our understanding of age-related sensory and motor function may help explain why the laryngeal function of some individual's declines and persists in others. Identifying what is underlying the neural changes in the aging larynx will lead to rejuvenation strategies, not only of laryngeal mucosa but also in laryngeal musculature, leveraging stem cells, tissue scaffolds, and growth factors.

Spasmodic dysphonia and vocal tremor are presently also largely treated at the end organ level. Frontiers in diagnosis and treatment of these disorders lie in clarifying the source of pathology in the central nervous system. Though largely thought of as motor disorders, sensory changes are known to play a significant role in these diseases. Advances in knowledge of the central neural pathology will be the nidus to change from symptomatic treatments to root level therapy and possibly cure.

Whether primary MTD and PVFD are entirely nonorganic is debatable. Little is known about sensory and motor dysfunction in this patient population. Voice therapy remains the primary treatment, but some patients respond quickly and dramatically, and others are recalcitrant to treatment. Viewing primary MTD and PVFD as neurolaryngological disorders may help explain the spectrum of disease and potentially direct therapy to motor or sensory anomalies if they are present. 
Laryngeal sensory neuropathic cough is presently a diagnosis of exclusion. Advances in sensory electrodiagnostic testing in the larynx will lead to more concrete understanding of this poorly understood problem. Additionally, refined central diagnostics and functional imaging of the brain may hold the key to knowledge about this diagnosis.

\section{Conclusion}

The nature of neurolaryngology is rooted in the complex interface of the larynx and its central and peripheral neural input. Thus it is no surprise that our understanding, diagnosis, and treatment of neurolaryngological disorders lags behind other aspects of laryngology, such as benign and malignant neoplasms, phonotraumatic lesions, and inflammatory disorders of the larynx. Advances in knowledge of both the efferent and the afferent neural physiology of the larynx will lead to a paradigm shift from largely treated at the end organ to diagnostic and therapeutic modalities at the primary source of the disorder.

The immaturity of neurolaryngology can very well be advantageous, as it allows for current and future researchers to glean from the advances in neurology and neurosurgery. Recent work in neural stem cell transplants and laser exposure of gold nanorods to stimulate neuronal cell growth may be applicable, saving our field decades of benchwork. The development of new neural interfaces between the brain and peripheral nerves or the use of organic conductive bioelectrodes may prove to be the key for restoring motor function of the larynx. On the genetics front, current work on molecular target discovery for neural repair may 1 day make surgical repair of paralyzed vocal folds obsolete.

Disclosure Michael M. Johns, Allen D. Hillel, and Adam M. Klein declare that they have no conflict of interest. 